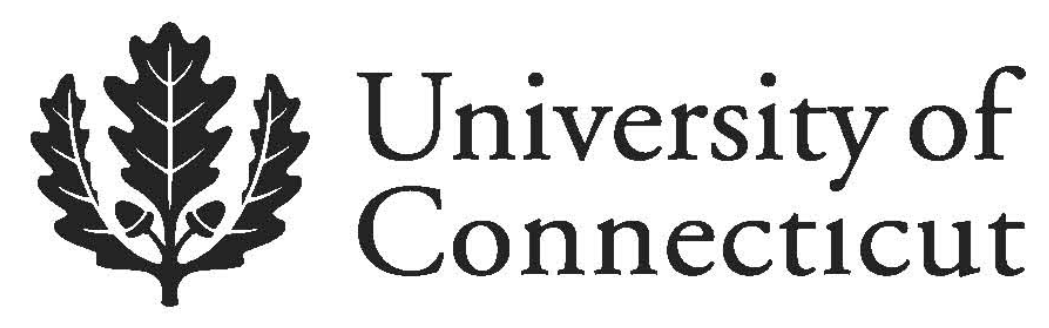

Department of Economics Working Paper Series

\title{
Information Concentration in Common Value Environments
}

Vlad Mares

Northwestern University

Mikhael Shor

University of Connecticut

Working Paper 2012-23

November 2008

365 Fairfield Way, Unit 1063

Storrs, CT 06269-1063

Phone: (860) 486-3022

Fax: (860) 486-4463

http://www.econ.uconn.edu/

This working paper is indexed on RePEc, http://repec.org 


\title{
Information Concentration in Common Value Environments *
}

\author{
Vlad Mares \\ Kellogg School of Management \\ Northwestern University \\ v-mares@kellogg.northwestern.edu
}

\author{
Mikhael Shor \\ Owen Graduate School of Management \\ Vanderbilt University \\ mike.shor@owen.vanderbilt.edu
}

This version: November 2008

\begin{abstract}
We consider how information concentration affects a seller's revenue in common value auctions. The common value is a function of $n$ random variables partitioned among $m \leq n$ bidders. For each partition, the seller devises an optimal mechanism. We show that, whenever the value function allows scalar sufficient statistics for each player's signals, the mechanism design problem is well-defined. Additionally, whenever a common regularity condition is satisfied, a coarser partition always reduces revenues. In particular, any merger or collusion among bidders reduces revenue.
\end{abstract}

JEL Classification Numbers: D44, L41, C72, D82

Key Words: information concentration, industry concentration, mechanism design, common-value auctions

${ }^{*}$ The authors would like to thank Luciano de Castro, Luke Froeb, Paul Klemperer, Rich McLean, and Rakesh Vohra, for valuable comments and suggestions. 


\section{Introduction}

Consider a seller of an indivisible item facing several potential buyers, each with some information about the object's common value. How would a decision by a group of buyers to centralize their information and decision-making authority impact the seller's revenue? While the question is of obvious interest to auctioneers considering allowing bidding syndicates and to policy governing mergers and collusion, these environments remain largely unmodeled. Providing a general result on the revenue impact of information concentration is the purpose of this manuscript.

A growing literature is devoted to determining how access to more informative signals changes behavior in allocation problems (Matthews 1984, Persico 2000, Athey and Levin 2001, Bergemann and Valimaki 2002, Mares and Harstad 2003). Since these approaches generally define "more informative" in terms of some order over scalar random variables, they permit two buyers working together to possess anything from only a slightly more informative signal than each of them had individually to nearly perfect information about the object's value. Another strand of literature finds that a more concentrated industry, obtained by removing a bidder and his information, is less profitable even when the auctioneer reacts with an optimal mechanism (Bulow and Klemperer 1996). However, this approach conflates the role of industry concentration with information concentration.

In contrast, our approach keeps the total amount of information constant while concentrating its allocation among fewer bidders. The centralization of two buyers' signals would simply have the joint entity with two signals. Since the new entity possesses a multi-dimensional signal, this calls into question the existence of equilibria in auctions (Jackson 2005) and of incentive-compatible mechanisms in general (Armstrong and Rochet 1999). Several authors have adopted models of bidding with multidimensional signals while imposing symmetry (Goeree and Offerman 2002, DeBrock and Smith 1983, Mares and Shor 2008). Yet, symmetric models are particularly ill-suited to modeling mergers or collusion as even an a priori symmetric industry will not be so following a merger 1 Without symmetry, simple auction mechanisms need not be optimal. In this paper, we provide the auctioneer full strategic latitude in the choice of mechanisms

In our model, the object's value is a function of $n$ independent (but not necessarily identically distributed) signals, which are allocated among $m \leq n$ bidders. We adopt a mechanism design approach, allowing the seller to select an optimal mechanism for each allocation of signals among bidders. We require that each bidder's vector of signals allows a scalar sufficient statistic, a condition satisfied by commonly-analyzed models, including additively separable and maximum or minimum value auctions (Mares and Shor 2008, Bikhchandani and Riley 1991, Bulow and Klemperer 2002, Krishna and Morgan 1997). Our central result is that a coarser partition of information among bidders always results in reduced revenue for the seller. This implies that all mergers reduce the auctioneer's revenue, even those that make an industry more symmetric by aggregating smaller bidders.

To approach the mechanism design problem in asymmetric, multidimensional-signals contexts, we provide a result that significantly simplifies the problem. Whenever a scalar sufficient summary statistic exists for each player's signals, every incentive-compatible mechanism has a revenue-equivalent incentive-compatible scalar mechanism that requires only scalar reports. We offer a constructive proof of this result in a general environment. This allows a broad class of problems to be analyzed in the Myerson (1981) framework, and provides sufficient conditions for the existence of incentive-compatible mechanisms.

\footnotetext{
${ }^{1}$ Common value auctions appear especially sensitive to asymmetries. Bikhchandani (2006) notes that even vanishing asymmetries can lead an advantaged bidder to win the auction with probability 1. Klemperer (1998) provides discussion of this result in relation to the FCC Spectrum auctions.
} 
In what follows, we describe a model of common-value environments that allows for arbitrary partitions of signals among bidders. We first present our result on the existence of optimal scalar mechanisms, which extends the definition of virtual valuations to these contexts. Then, we present our main result, that coarser partitions of information in regular allocation problems decreases revenue. We demonstrate these results for a class of value functions and conclude with policy implications and suggestions for future research.

\section{Model}

A seller has one indivisible object and faces $m$ risk-neutral buyers. Consider a collection of $n$ independent random variables, $\left(X_{1}, \ldots, X_{n}\right)$, where $X_{i}$ has density $f_{i}$, distribution $F_{i}$, and typical element $x_{i} \in\left[\underline{z}_{i}, \bar{z}_{i}\right] \subset$ $\Re 2^{2}$ An information profile $A=\left(A_{1}, . ., A_{m}\right)$ is a partition of the index set $\{1, \ldots, n\}$, with the interpretation that $\mathbf{S}_{i} \equiv\left\{X_{k}\right\}_{k \in A_{i}}$ describes bidder $i$ 's private information with typical element $s_{i} \in \Delta_{i} \equiv \prod_{k \in A_{i}}\left[\underline{z}_{k}, \bar{z}_{k}\right] \subset$ $\Re^{\left|A_{i}\right|}$. A vector of realizations of private information is denoted $\mathbf{s}=\left(\mathbf{s}_{i}, \mathbf{s}_{-i}\right)=\left(\mathbf{s}_{1}, \ldots, \mathbf{s}_{m}\right)$.

Bidder $i$ 's valuation for the object is given by $V_{i}(\mathbf{s})$ which is increasing in $\mathbf{s}_{i}$ and nondecreasing in $\mathbf{s}_{-i}$. This model generalizes the standard symmetric auction model. The special case of each bidder possessing one identically distributed signal is obtained when $m=n, A_{i}=\{i\}$, and $F_{i} \equiv F$.

The seller constructs a mechanism, $\eta=\left(p_{i}(\mathbf{s}), \xi_{i}(\mathbf{s})\right)$, which determines allocation probabilities and payments based on buyers' reports, s. Define information profile $A^{\prime}$ as coarser than information profile $A$, if, for each $a \in A$ there exists an $a^{\prime} \in A^{\prime}$ such that $a \subseteq a^{\prime}$. In particular, consider a simple merger between bidders 1 and 2, so that the single entity shares all information and places a single bid. By redistributing all of bidder 2's information towards 1, the post-merger information profile, $A^{\prime}=\left\{A_{1} \cup A_{2}, A_{3}, \ldots, A_{n}\right\}$ is coarser than the pre-merger profile $A=\left\{A_{1}, A_{2}, A_{3}, \ldots, A_{n}\right\}$.

\section{Scalar Mechanisms}

We will show when each player's type is multidimensional but admits a sufficient statistic, the search for optimal mechanisms is simplified. Scalar mechanisms, which require only the report of the sufficient statistic, instead of the full vector describing the private information, are natural candidates in such settings. Their reliance on scalar private reports for the allocation and payment problem reduces the dimensionality of the message space under consideration. We will show that they can replicate the revenue properties of their more complex counterparts.

Assume that, for player 1, the value functions admit sufficient statistic representations. Formally, there exists a function $\phi_{1}: \Delta_{1} \rightarrow \Re$ which satisfies, for all $j$ and any $\mathbf{s}_{-1}$,

$$
V_{j}\left(\mathbf{s}_{1}, \mathbf{s}_{-1}\right) \geq V_{j}\left(\mathbf{s}_{1}^{\prime}, \mathbf{s}_{-1}\right) \Leftrightarrow \phi_{1}\left(\mathbf{s}_{1}\right) \geq \phi_{1}\left(\mathbf{s}_{1}^{\prime}\right)
$$

We define two types $\mathbf{s}_{1}$ and $\mathbf{s}_{1}^{\prime}$ as equivalent if $\phi_{1}\left(\mathbf{s}_{1}\right)=\phi_{1}\left(\mathbf{s}_{1}^{\prime}\right)$.

For every mechanism $\eta=\left(p_{i}\left(\mathbf{s}_{i}, \mathbf{s}_{-i}\right), \xi_{i}\left(\mathbf{s}_{i}, \mathbf{s}_{-i}\right)\right)$ we can construct a scalar mechanism

\footnotetext{
${ }^{2}$ These random variables need not be identically distributed, but we do require independence. Otherwise, the results of Crémer and McLean (1988) and McAfee and Reny (1992) would imply full revenue extraction, trivially eliminating any impacts information concentration may have on revenue as long as two buyers remain.
} 
$\eta^{\prime}=\left(p_{i}^{\prime}\left(\mathbf{s}_{i}, \mathbf{s}_{-i}\right), \xi_{i}^{\prime}\left(\mathbf{s}_{i}, \mathbf{s}_{-i}\right)\right)$ where

$$
\begin{aligned}
& p_{j}^{\prime}\left(\phi_{1}\left(\mathbf{s}_{1}\right), \mathbf{s}_{-1}\right)=\int p_{j}\left(\mathbf{t}_{1}, \mathbf{s}_{-1}\right) f_{1}\left(\mathbf{t}_{1} \mid \phi_{1}\left(\mathbf{t}_{1}\right)=\phi_{1}\left(\mathbf{s}_{1}\right)\right) d \mathbf{t}_{1}, \text { and } \\
& \xi_{j}^{\prime}\left(\phi_{1}\left(\mathbf{s}_{1}\right), \mathbf{s}_{-1}\right)=\int \xi_{j}\left(\mathbf{t}_{1}, \mathbf{s}_{-1}\right) f_{1}\left(\mathbf{t}_{1} \mid \phi_{1}\left(\mathbf{t}_{1}\right)=\phi_{1}\left(\mathbf{s}_{1}\right)\right) d \mathbf{t}_{1} .
\end{aligned}
$$

for every $j$ and $\mathbf{s}_{1}$. The scalar mechanism, $\eta^{\prime}$, averages the allocation probabilities and payment functions over the set of equivalent types. Two equivalent types $\mathbf{s}_{1}$ and $\mathbf{s}_{1}^{\prime}$ will receive the asset with the same probability $p_{1}^{\prime}\left(\phi_{1}\left(\mathbf{s}_{1}\right), \mathbf{s}_{-1}\right)$ and face the same payment $\xi_{1}^{\prime}\left(\phi_{1}\left(\mathbf{s}_{1}\right), \mathbf{s}_{-1}\right)$. Furthermore, from player $j$ 's perspective, the allocation probability and payment are invariant across equivalent types of player 1 . In essence, the mechanism $\eta^{\prime}$ requires only a report of the sufficient statistic of player 1's private information.

We show that mechanisms $\eta$ and $\eta^{\prime}$ are revenue equivalent. Additionally, if $\eta$ is incentive compatible, then so is $\eta^{\prime}$, so any mechanism has an associated revenue-equivalent scalar mechanism.

Theorem 1. For any set of value functions which admit sufficient statistics for player $i$, and any incentivecompatible mechanism $\eta$, we can construct an incentive-compatible scalar mechanism $\eta^{\prime}$ which has the same revenue as $\eta$ but has probability and payment functions which depend only on the sufficient statistic $\phi_{i}$ of the private information of player $i$.

The theorem addresses a single bidder whose information can be summarized via a sufficient statistic. Its extension to instances where multiple or even all player's private information can be captured by scalars is straightforward, requiring repeated application of the theorem. The theorem allows the search for optimal mechanisms to consider only optimal scalar mechanisms.

The result guarantees that a scalar mechanism exists in the set of all optimal mechanisms for this problem. Scalar mechanisms are appealing as they are simpler and easier to implement than mechanisms requiring multiple reports from each bidder, especially when different bidders are asked for different quantities of reports. If we wish to identify the maximum revenue obtainable by the seller, we need only identify an optimal scalar mechanism. The next section exploits this property by establishing, for a certain class of value functions, a strong order among revenues generated by different information partitions.

\section{Information Concentration}

We now investigate the impact of information concentration on the seller's revenues. We will treat information concentration as a redistribution of the available information among bidders. Intuitively, one can think of a process of coalition formation whereby bidders "buy out" their competitors, gaining access to their private information. Each such merger among bidders transforms the information profile, $A$, into a coarser information profile, $A^{\prime}$.

For the remainder of the manuscript, we concentrate on pure common value auctions, $V_{i}(\cdot) \equiv V(\cdot)$, where the value of the object is the same for each bidder under every information profile 3 We place the following assumptions on $V$ :

Assumption 1. $V$ is increasing and weakly supermodular: $\partial_{i} V>0$ and $\partial_{i j} V \geq 0$ for all $i, j$.

Assumption 2. $V$ admits sufficient statistic representations for all players and information profiles.

\footnotetext{
${ }^{3}$ To focus on the role of information concentration, the pure common value auction implies that mergers do not have value-improving synergies.
} 
Consider the case where two bidders, each with one signal, combine their information. As a first step, we derive characteristics of the distribution of this summary statistic. Let $X_{i}$ be two independent random variables with distribution and density functions $F_{i}$ and $f_{i}$ and typical element $x_{i} \in\left[\underline{z}_{i}, \bar{z}_{i}\right]$. Denote the survival function by $\bar{F}_{i}=1-F_{i}$. Let their summary statistic, $\phi(\cdot, \cdot):\left[\underline{z}_{1}, \bar{z}_{1}\right] \times\left[\underline{z}_{2}, \bar{z}_{2}\right] \rightarrow \Re$, be strictly increasing in both arguments, and admit continuous partial derivatives. Denote by $F_{\phi}, f_{\phi}$, and $\bar{F}_{\phi}$ the distribution, density, and survival function of the random variable $\phi\left(X_{1}, X_{2}\right)$.

Since sufficient statistics are uniquely defined only up to a monotone transformation, we arbitrarily select one set of representations. For any $A_{i} \subset\{1, \ldots, n\}$ pick a $\phi^{A_{i}}: \Re^{\left|A_{i}\right|} \rightarrow \Re$ such that

$$
V\left(\mathbf{s}_{i}, \mathbf{s}_{-i}\right) \geq V\left(\mathbf{s}_{i}^{\prime}, \mathbf{s}_{-i}\right) \Leftrightarrow \phi^{A_{i}}\left(\mathbf{s}_{i}\right) \geq \phi^{A_{i}}\left(\mathbf{s}_{i}^{\prime}\right)
$$

for all $\mathbf{s}_{i}=\left\{x_{k}\right\}_{k \in A_{i}}$ and $\mathbf{s}_{i}^{\prime}$. The set of sufficient statistics will be denoted

$$
\Phi=\left\{\phi^{A_{i}} \mid A_{i} \in 2^{A}\right\} .
$$

It will be convenient to refer to value functions defined over the space of sufficient statistics, rather than the elementary signals. Define $V^{\phi^{A_{i}}}: \Re^{\sum_{j \neq i}\left|A_{j}\right|+1} \rightarrow \Re$

$$
V^{\phi^{A_{i}}}\left(t_{i}, \mathbf{s}_{-i}\right)=V\left(\mathbf{s}_{i}, \mathbf{s}_{-i}\right)
$$

whenever there exists an $\mathbf{s}_{i}$ such that $\phi^{A_{i}}\left(\mathbf{s}_{i}\right)=t_{i}$. This is the value function when bidder $i$ 's (possibly multidimensional) information is replaced with its scalar sufficient statistic. Iterating this procedure, we define for every information profile $A=\left\{A_{1}, . ., A_{m}\right\}$, the function $V^{A}: \Re^{|A|} \rightarrow \Re$

$$
V^{A}\left(t_{1}, . ., t_{m}\right)=V\left(\mathbf{s}_{1}, . ., \mathbf{s}_{m}\right)
$$

if, for all $i \in\{1, \ldots, m\}, \phi^{A_{i}}\left(\mathbf{s}_{i}\right)=t_{i}$. We denote by $t_{-i}$ the vector of sufficient statistics excluding bidder $i$. It is straightforward to show that the functions $V^{A}$ inherit several properties of the function $V$. In particular, $V^{A}$ is increasing and weakly supermodular, $\partial_{i} V^{A}>0$ and $\partial_{i j} V^{A} \geq 0$.

An allocation problem in this context is a collection $\left(n, V,\left\{X_{i}\right\}_{i=1}^{n},\left\{F_{i}\right\}_{i=1}^{n}, \Phi\right)$. We require the following regularity condition for our main result.

Definition. An allocation problem is regular if for any information profile $A=\left\{A_{1}, . ., A_{m}\right\}$ the function

$$
g_{i}^{A}\left(t_{i}, t_{-i}\right)=\frac{\bar{F}_{\phi^{A_{i}}}\left(t_{i}\right)}{f_{\phi^{A_{i}}}\left(t_{i}\right)} \partial_{i} V^{A}\left(t_{i}, t_{-i}\right),
$$

is nonincreasing in $t_{i}$.

By Assumption $1, g_{i}^{A}(\cdot)$ is nondecreasing in $t_{j}, j \neq i$. Define

$$
H_{i}^{A}\left(t_{i}, t_{-i}\right)=V^{A}\left(t_{i}, t_{-i}\right)-g_{i}^{A}\left(t_{i}, t_{-i}\right)
$$

as the virtual valuations under a scalar mechanism. Our regularity condition implies that these virtual valuations are increasing, analogous to the condition in Myerson (1981). We now state our main result.

Theorem 2. For all regular allocation problems, coarser profiles will lead to lower revenues. 
For a sketch of the proof, consider a simple merger where bidder 1 and 2's information is centralized under the control of a new bidder $c$. Let

$$
A=\left\{A_{1}, A_{2}, \ldots, A_{n}\right\}
$$

be the pre-merger information profile and

$$
A^{\prime}=\left\{A_{c}, A_{3}, \ldots, A_{n}\right\}
$$

represent post-merger, where $A_{c}=A_{1} \cup A_{2}$.

We show that an incentive-compatible mechanism exists in the pre-merger case that is revenue equivalent to the optimal mechanism post-merger. Effectively, it treats bidders 1 and 2 as if they had merged, even though they do not share information. In the optimal scalar mechanism under $A^{\prime}$, each bidder $i \geq 3$ submits a scalar report, $t_{i}$, and bidder $c$ submits a scalar report $t_{c}$. The proof follows three steps. First, we characterize the optimal post-merger mechanism $\mu^{A^{\prime}}$. Second, we construct a mechanism $\omega$ for the information profile $A$, which treats bidders 3 through $n$ identically as $\mu^{A^{\prime}}$ but allocates to bidder 2 whenever bidder $c$ would have won under $\mu^{A^{\prime}}$. Finally, we show that $\omega$ is incentive compatible and revenue-equivalent to $\mu^{A^{\prime}}$. Since $\omega$ is revenue-dominated by $\mu^{A}$, the optimal mechanism for information profile $A$, we establish the desired result.

While we define a regular allocation problem as requiring the monotonicity of $g_{i}^{A}(\cdot)$ for every information profile, it is possible that monotonicity is satisfied only for some information profile $A$. In this case, we still conclude that the coarsening of $A$ leads to lower revenues. Additionally, if the optimal mechanism under every information profile requires each buyer to have a strictly positive allocation probability, then a coarser profile leads to strictly lower profits. This assures that $\omega$ and $\mu^{A}$ are not equivalent, as no buyer is irrelevant.

The central argument behind our revenue comparisons in Theorem 2 is quite general. Starting from an optimal mechanism under a concentrated profile, we construct an allocation-equivalent mechanism for a profile where one of the bidding rings is dissolved into its component members. The new mechanism allocates to one representative member whenever the old mechanism allocated towards the ring, and does not otherwise modify allocations. This generates the same surplus as the old mechanism, but is not necessarily optimal in the new environment. Our argument relies crucially on the independence assumption since this guarantees that the new mechanism satisfies the IR and IC constraints for the representative of the ring.

The requirement that a scalar sufficient statistic exists is also a critical assumption. Our argument assumes the existence of an optimal mechanism under the concentrated profile. While there is no guarantee that such an optimal mechanism exists generically, the scalarization assumption and regularity condition are sufficient for a constructive proof, using the approach of Myerson (1981) ${ }^{4}$

\section{Discussion}

Information concentration decreases revenue, even when the auctioneer can respond optimally to the new industry structure. This is similar in spirit to the result of Waehrer and Perry (2003) who find, in symmetric private value environments, revenues are decreasing even if the auctioneer adjusts his mechanism optimally

\footnotetext{
${ }^{4}$ Note also that we represent the transition from concentrated to non-concentrated environments at a very abstract level. Any bidding ring can be dissolved into its component members. This has the flavor of ex-post implementation in its incentive compatibility requirement for the representative of the ring. A recent paper by Bikhchandani (2006) shows that in multidimensional problems a single-crossing condition akin to scalarization is required for the existence ex-post implementation.
} 
to account for less competitive profiles. In common value environments, our result complements that of Bulow and Klemperer (1996), though with one important distinction. Bulow and Klemperer compare an optimal mechanism under a less competitive scenario to a standard auction with more bidders. A no-reserve English auction with $N+1$ bidders revenue-dominates any mechanism with $N$ bidders, when signals are independent. This result has been commonly interpreted as stating that the competition effect outweighs any mechanism design variable the auctioneer could introduce. However, $N$ bidders are also collectively less informed than $N+1$ bidders, diluting the argument of the strength of the competitive effect. Our results show that, keeping the informational content constant, we can isolate a competitive effect which reduces the seller's revenues even if we allow the seller full latitude in the choice of mechanisms.

Recently, Mares and Shor (2008) have identified a revenue effect of concentration in wallet games, where the value is equal to the sum of the signals and signals have log-concave density. They consider only symmetric industry profiles and first and second price auctions. For example, six firms each with one signal yields higher revenue for the auctioneer than three firms each with two signals. With symmetry, revenue equivalence allows the mechanism design issue to be sidestepped. We can apply our theorem to generalize their result to all concentrated profiles. Consider the following value function:

$$
V\left(X_{1}, \ldots, X_{n},\right)=\sum X_{k}
$$

where each $X_{i}$ has log-concave density $f_{i}$ and distribution $F_{i}$. Clearly, the function is increasing in all of its arguments and is (weakly) supermodular. Further, $\phi^{A_{i}}\left(\mathbf{s}_{i}\right)=\sum_{k \in A_{i}}\left(X_{k}\right)$ is a sufficient statistic for $\mathbf{s}_{i}$. Thus, we can write the value function over summary statistics,

$$
V^{A}\left(t_{i}, t_{-i}, \alpha\right)=t_{i}+\sum_{j \neq i} t_{j}
$$

To check our regularity condition, we must establish the monotonicity in $t_{i}$ of

$$
g_{i}^{A}\left(t_{i}, t_{-i}\right)=\frac{\bar{F}_{\phi^{A} i}\left(t_{i}\right)}{f_{\phi^{A}}\left(t_{i}\right)} \partial_{i} V^{A}\left(t_{i}, t_{-i}\right)
$$

Consider $X$ and $Y$ independent variables with $\log$-concave densities $f_{X}$ and $f_{Y}$, which is satisfied by most common distributions (Bagnoli and Bergstrom 2005). This implies that $X$ and $Y$ have log-concave survival functions $\bar{F}_{X}$ and $\bar{F}_{Y}$ (Prékopa 1971, 1973). Therefore, $Z=X+Y$ has a log-concave distribution and survival function. In particular, $\frac{\bar{F}_{Z}\left(t_{i}\right)}{f_{Z}\left(t_{i}\right)}$ is non-increasing. Since $\partial_{i} V^{A}\left(t_{i}, t_{-i}\right)=1$, our regularity condition is satisfied.

Corollary 2.1. In the wallet game, coarser information profiles lead to lower revenues when signals have log-concave distributions.

In order to resolve the potential lack of equilibria arising from multi-dimensional signals (Jackson 2005), researchers have focused on a few specific models for which equilibria have been analytically identified (e.g., Goeree and Offerman 2002, DeBrock and Smith 1983, Mares and Shor 2008). Our results suggest that researchers need not be restricted to these specialized models. Any model that allows for scalar sufficient statistics of each bidder's information can be analyzed in the familiar framework of mechanism design with a scalar message space. The scalarization result allows us to consider the general question of the impact of information concentration. 
Our results have significant applications for antitrust. In traditional price-setting markets, mergers among smaller, higher-priced firms can reduce average prices by shifting quantity to lower-priced firms post-merger (Werden and Froeb 1994). Similarly, in private value auctions, the auctioneer prefers to face symmetric bidders, holding the average value distribution constant (Cantillon 2008). Even profitable mergers of relatively weak competitors can lead to higher revenues for the auctioneer 5 This benefit of concentration among smaller market players has been suggested as an efficiency defense in mergers (Dagen and Richards 2006), and several regulators have appeared sensitive to these claims.6 ${ }^{6}$ Klemperer (2005) argues that a positive view of joint bidding is largely rooted in a misinterpretation of past research, and suggests, in line with our results, that it is likely to be harmful for the seller. Yet, largely due to conjectures that mergers in common-value auctions can increase revenue (e.g., Krishna and Morgan 1997) and lack of suitable theoretical evidence to the contrary, some have called for regulatory restraint, advising regulators to err on the side of less oversight when considering common-value environments (Froeb and Shor 2005).

Should an auctioneer facing several bidders ever allow a consortium to form or bidders to merge? Perhaps synergies not considered here can have a significant enough positive impact, but the concentration of information, alone, cannot be of benefit to the auctioneer. Our results indicate an unambiguous revenue-reducing impact of information concentration.

\footnotetext{
${ }^{5}$ Thomas (2004) demonstrates how "a profitable efficiency increasing merger of two relatively small firms creates a stronger competitor that can cause the expected price to fall [in procurement settings], despite the resulting increase in market concentration." (p. 688).

${ }^{6}$ For example, see the comments of Andrew R. Dick, former Acting Chief of the policy section at the DOJ Antitrust Division, J. Mark Gidley, Assistant Attorney General for Antitrust, and David T. Scheffman, former Director of the FTC's Bureau of Competition, who all suggest that asymmetry-reducing mergers can provide market efficiencies (FTC/DOJ 2004).
} 


\section{References}

Armstrong, Mark, and Jean-Charles Rochet (1999): "Multi-dimensional screening: A user's guide," European Economic Review, 43(4-6), 959-979.

Athey, Susan, And Jonathan Levin (2001): "The Value of Information in Monotone Decision Problems," Working Paper, MIT.

Bagnoli, Mark, And Ted Bergstrom (2005): "Log-Concave Probability and its Applications," Economic Theory, 26(2), 445-469.

Bergemann, Dirk, and Juuso Valimaki (2002): "Information Acquisition and Efficient Mechanism Design," Econometrica, 70(3), 1007-1033.

Bikhchandani, Sushil (2006): "Ex Post Implementation in Environments with Private Goods," Theoretical Economics, 1(3), 369-393.

Bikhchandani, Sushil, And John Riley (1991): "Equilibria in Open Common Value Auctions," Journal of Economic Theory, 53(1), 101-130.

Bulow, Jeremy, and Paul Klemperer (1996): "Auctions versus Negotiations," American Economic Review, 86(1), 180-194.

(2002): "Prices and the Winner's Curse," RAND Journal of Economics, 33(1), 1-21.

Cantillon, Estelle (2008): "The Effect of Bidders' Asymmetries on Expected Revenue in Auctions," Games and Economic Behavior, 62(1), 1-25.

Crémer, Jacques, and Richard McLean (1988): "Full Extraction of the Surplus in Bayesian and Dominant Strategy Auctions," Econometrica, 56(6), 1247-1257.

Dagen, Richard, and Daniel Richards (2006): "Merger Theory and Evidence: The Baby-Food Case Reconsidered," Working Paper, Tufts University Department of Economics.

DeBrock, L., And J. Smith (1983): "Joint Bidding, Information Pooling, and the Performance of Petroleum Lease Auctions," Bell Journal of Economics, 14(2), 395-404.

Froeb, Luke, and Mikhael Shor (2005): "Auction Models," in Econometrics: Legal, Practical, and Technical Issues, ed. by John D. Harkider, pp. 225-246. American Bar Association Section of Antitrust Law.

FTC/DOJ (2004): “Joint Workshop on Merger Enforcement," February 17-19, Washington, D.C.

Goeree, Jacob K., And Theo Offerman (2002): "Efficiency in Auctions with Private and Common Values: An Experimental Study," American Economic Review, 93(3), 625-643.

Jackson, Matthew O. (2005): "The Non-Existence of Equilibrium in Vickrey, Second-Price, and English Auctions," Working Paper, California Institute of Technology.

Klemperer, Paul (1998): "Auctions with Almost Common Values: The 'Wallet Game' and its Applications," European Economic Review, 42(3-5), 757-769.

_ (2005): "Bidding Markets," UK Competition Commission Occasional Paper No. 1.

Krishna, Vijay, and John Morgan (1997): “(Anti-) Competitive Effects of Joint Bidding and Bidder Restrictions," Working Paper, Penn State University and Princeton University.

Mares, Vlad, and Ronald M. Harstad (2003): "Private information revelation in common-value auctions," Journal of Economic Theory, 109(2), 264-282. 
Mares, Vlad, And Mikhael Shor (2008): "Industry Concentration in Common Value Auctions," Economic Theory, 35, 37-56.

Matthews, Steven A. (1984): "Information Acquisition in Discriminatory Auctions," in Bayesian Models in Economic Theory, ed. by Marcel Boyer, and Richard E. Kihlstrom, pp. 181-207. Elsevier Science.

McAfee, R. Preston, and Philip J. Reny (1992): "The Competitive Effects of Mergers Between Asymmetric Firms," Correlated Information and Mechanism Design, 60(2), 395-421.

Myerson, R. (1981): "Optimal Auction Design," Mathematics of Operations Research, 6(1), 58-73.

Persico, Nicola (2000): "Information Acquisition in Auctions," Econometrica, 68(1), 135-148.

PRÉKOPA, ANDRÁs (1971): "Logarithmic concave measures with application to stochastic programming," ACTA Sci. Math. (Szeged), 32, 301-316.

- (1973): "On logarithmic concave measures and functions," ACTA Sci. Math. (Szeged), 34, 335-343.

Thomas, Charles J. (2004): "The Competitive Effects of Mergers Between Asymmetric Firms," International Journal of Industrial Organization, 22(5), 679-692.

Waehrer, Keith, and Martin K. Perry (2003): "The Effects of Mergers in Open-Auction Markets," RAND Journal of Economics, 34(2), 287-304.

Werden, Gregory J., And Luke M. Froeb (1994): "The Effects of Mergers in Differentiated Products Industries: Logit Demand and Merger Policy," Journal of Law, Economics, ES Organization, 10(2), 407426 . 


\section{Appendix}

\section{Proof of Theorem 1}

The proof requires three lemmas. Fix the player for whom the value functions admit sufficient statistics to be player 1 .

Lemma 1. Under any incentive compatible mechanism $\eta$, the expected payoff for equivalent types is equal.

Proof. For every mechanism $\eta=\left(p_{i}(\cdot), \xi_{i}(\cdot)\right)$ define

$$
\widetilde{V}_{j}\left(\mathbf{s}_{j} ; \mathbf{t}_{j}\right)=\int V_{j}\left(\mathbf{s}_{j}, \mathbf{s}_{-j}\right) p_{j}\left(\mathbf{t}_{j}, \mathbf{s}_{-j}\right) f_{-j}\left(\mathbf{s}_{-j}\right) d \mathbf{s}_{-j}
$$

and

$$
\widetilde{\xi}_{j}\left(\mathbf{t}_{j}\right)=\int \xi_{j}\left(\mathbf{t}_{j}, \mathbf{s}_{-j}\right) f_{-j}\left(\mathbf{s}_{-j}\right) d \mathbf{s}_{-j}
$$

Under mechanism $\eta$, the expected payoff for player $j$ who has information $\mathbf{s}_{j}$ and reports $\mathbf{t}_{j}$ is

$$
\widetilde{V}_{j}\left(\mathbf{s}_{j} ; \mathbf{t}_{j}\right)-\widetilde{\xi}_{j}\left(\mathbf{t}_{j}\right)
$$

The interim incentive compatibility constraint for player 1 is

$$
\widetilde{V}_{1}\left(\mathbf{s}_{1} ; \mathbf{s}_{1}\right)-\widetilde{\xi}_{1}\left(\mathbf{s}_{1}\right) \geq \widetilde{V}_{1}\left(\mathbf{s}_{1} ; \mathbf{t}_{1}\right)-\widetilde{\xi}_{1}\left(\mathbf{t}_{1}\right)
$$

for all $\mathbf{s}_{1}$ and $\mathbf{t}_{1}$. For two equivalent types $\mathbf{s}_{1}$ and $\mathbf{s}_{1}^{\prime}$ and any $\mathbf{t}_{1}$, we have by definition

$$
\widetilde{V}_{1}\left(\mathbf{s}_{1} ; \mathbf{t}_{1}\right)=\widetilde{V}_{1}\left(\mathbf{s}_{1}^{\prime} ; \mathbf{t}_{1}\right)
$$

and in particular

$$
\begin{aligned}
& \widetilde{V}_{1}\left(\mathbf{s}_{1} ; \mathbf{s}_{1}\right)=\widetilde{V}_{1}\left(\mathbf{s}_{1}^{\prime} ; \mathbf{s}_{1}\right), \\
& \widetilde{V}_{1}\left(\mathbf{s}_{1}^{\prime} ; \mathbf{s}_{1}^{\prime}\right)=\widetilde{V}_{1}\left(\mathbf{s}_{1} ; \mathbf{s}_{1}^{\prime}\right) .
\end{aligned}
$$

Substituting back into the incentive compatibility constraints we have

$$
\widetilde{V}_{1}\left(\mathbf{s}_{1} ; \mathbf{s}_{1}\right)-\widetilde{V}_{1}\left(\mathbf{s}_{1}^{\prime} ; \mathbf{s}_{1}^{\prime}\right)=\widetilde{V}_{1}\left(\mathbf{s}_{1} ; \mathbf{s}_{1}\right)-\widetilde{V}_{1}\left(\mathbf{s}_{1} ; \mathbf{s}_{1}^{\prime}\right) \geq \widetilde{\xi}_{1}\left(\mathbf{s}_{1}\right)-\widetilde{\xi}_{1}\left(\mathbf{s}_{1}^{\prime}\right)
$$

and

$$
\widetilde{\xi}_{1}\left(\mathbf{s}_{1}\right)-\widetilde{\xi}_{1}\left(\mathbf{s}_{1}^{\prime}\right) \geq \widetilde{V}_{1}\left(\mathbf{s}_{1}^{\prime} ; \mathbf{s}_{1}\right)-\widetilde{V}_{1}\left(\mathbf{s}_{1}^{\prime} ; \mathbf{s}_{1}^{\prime}\right)=\widetilde{V}_{1}\left(\mathbf{s}_{1} ; \mathbf{s}_{1}\right)-\widetilde{V}_{1}\left(\mathbf{s}_{1}^{\prime} ; \mathbf{s}_{1}^{\prime}\right)
$$

which yields

$$
\widetilde{V}_{1}\left(\mathbf{s}_{1} ; \mathbf{s}_{1}\right)-\widetilde{\xi}_{1}\left(\mathbf{s}_{1}\right)=\widetilde{V}_{1}\left(\mathbf{s}_{1}^{\prime} ; \mathbf{s}_{1}^{\prime}\right)-\widetilde{\xi}_{1}\left(\mathbf{s}_{1}^{\prime}\right)=\widetilde{V}_{1}\left(\mathbf{s}_{1} ; \mathbf{s}_{1}^{\prime}\right)-\widetilde{\xi}_{1}\left(\mathbf{s}_{1}^{\prime}\right) .
$$

Consider the mechanism $\eta^{\prime}$ as defined by equations (1) and (2).

Lemma 2. If mechanism $\eta$ is incentive compatible, then $\eta^{\prime}$ is an incentive compatible mechanism.

Proof. Define $f_{\phi_{1}}$ as the density of $\phi_{1}\left(\mathbf{s}_{1}\right)$. Also, for every $\mathbf{s}_{1}$, such that $\phi_{1}\left(\mathbf{s}_{1}\right)=\alpha$, define by

$$
V_{i}^{\phi_{1}}\left(\alpha ; \mathbf{s}_{-1}\right)=V_{i}\left(\mathbf{s}_{1}, \mathbf{s}_{-1}\right)
$$


the parametrization of $i$ 's value function based on the sufficient statistic's value. Since $\eta$ is a mechanism, we have $p_{i}\left(\mathbf{s}_{1}, \mathbf{s}_{-1}\right) \geq 0$ and $\sum p_{i}\left(\mathbf{s}_{1}, \mathbf{s}_{-1}\right) \leq 1$ for every $i$ and every $\left(\mathbf{s}_{1}, \mathbf{s}_{-1}\right)$. This implies by integration over the set of equivalent types that for $i$ and $\mathbf{s}_{1}$,

$$
\int p_{i}\left(\mathbf{t}_{1}, \mathbf{s}_{-1}\right) f_{1}\left(\mathbf{t}_{1} \mid \phi_{1}\left(\mathbf{t}_{1}\right)=\phi_{1}\left(\mathbf{s}_{1}\right)\right) d \mathbf{t}_{1} \geq 0 \Leftrightarrow p_{i}^{\prime}\left(\phi_{1}\left(\mathbf{s}_{1}\right), \mathbf{s}_{-1}\right) \geq 0
$$

and similarly

$$
\sum p_{i}^{\prime}\left(\phi_{1}\left(\mathbf{s}_{1}\right), \mathbf{s}_{-1}\right) \leq 1
$$

The interim incentive compatibility condition for player $j$ under mechanism $\eta$ is

$$
\widetilde{V}_{j}\left(\mathbf{s}_{j} ; \mathbf{s}_{j}\right)-\widetilde{\xi}_{j}\left(\mathbf{s}_{j}\right) \geq \widetilde{V}_{j}\left(\mathbf{s}_{j} ; \mathbf{t}_{j}\right)-\widetilde{\xi}_{j}\left(\mathbf{t}_{j}\right)
$$

for all $\mathbf{s}_{j}$ and $\mathbf{t}_{j}$. For bidder $j \neq 1$,

$$
\begin{aligned}
\widetilde{\xi}_{j}\left(\mathbf{t}_{j}\right) & =\int \xi_{j}\left(\mathbf{t}_{j}, \mathbf{s}_{1}, \mathbf{s}_{-1 j}\right) f_{1}\left(\mathbf{s}_{1}\right) f_{-1 j}\left(\mathbf{s}_{-1 j}\right) d \mathbf{s}_{-j} \\
& =\int f_{-1 j}\left(\mathbf{s}_{-1 j}\right) \int f_{\phi_{1}}(\alpha) \int \xi_{j}\left(\mathbf{t}_{j}, \mathbf{s}_{1}, \mathbf{s}_{-1 j}\right) f_{1}\left(\mathbf{s}_{1} \mid \phi_{1}\left(\mathbf{s}_{1}\right)=\alpha\right) d \mathbf{s}_{1} d \alpha d \mathbf{s}_{-1 j} \\
& =\iint \xi_{j}^{\prime}\left(\alpha, \mathbf{t}_{j}, \mathbf{s}_{-1 j}\right) f_{-1 j}\left(\mathbf{s}_{-1 j}\right) f_{\phi_{1}}(\alpha) d \mathbf{s}_{-1 j} d \alpha \\
& =\widetilde{\xi}_{j}^{\prime}\left(\mathbf{t}_{j}\right) .
\end{aligned}
$$

Similarly, for all $\mathbf{s}_{j}$ and $\mathbf{t}_{j}$,

$$
\begin{aligned}
\widetilde{V}_{j}\left(\mathbf{s}_{j} ; \mathbf{t}_{j}\right) & =\int V_{j}\left(\mathbf{s}_{j}, \mathbf{s}_{1}, \mathbf{s}_{-1 j}\right) p_{j}\left(\mathbf{t}_{j}, \mathbf{s}_{1}, \mathbf{s}_{-1 j}\right) f_{1}\left(\mathbf{s}_{1}\right) f_{-1 j}\left(\mathbf{s}_{-1 j}\right) d \mathbf{s}_{-j}= \\
& =\iint V_{j}^{\phi_{1}}\left(\alpha ; \mathbf{s}_{j}, \mathbf{s}_{-1 j}\right) p_{j}^{\prime}\left(\alpha, \mathbf{t}_{j}, \mathbf{s}_{-1 j}\right) f_{-1 j}\left(\mathbf{s}_{-1 j}\right) f_{\phi_{1}}(\alpha) d \mathbf{s}_{-1 j} d \alpha \\
& =\widetilde{V}_{j}^{\prime}\left(\mathbf{s}_{j} ; \mathbf{t}_{j}\right) .
\end{aligned}
$$

Substituting the identical terms into the incentive compatibility constraint we get

$$
\widetilde{V}_{j}^{\prime}\left(\mathbf{s}_{j} ; \mathbf{s}_{j}\right)-\widetilde{\xi}_{j}^{\prime}\left(\mathbf{s}_{j}\right) \geq \widetilde{V}_{j}^{\prime}\left(\mathbf{s}_{j} ; \mathbf{t}_{j}\right)-\widetilde{\xi}_{j}^{\prime}\left(\mathbf{t}_{j}\right)
$$

for all $\mathbf{s}_{j}$ and $\mathbf{t}_{j}$ which indicates that mechanism $\eta^{\prime}$ is incentive compatible for player $j$.

Finally, we need to show that player 1's incentive compatibility constraint is satisfied under mechanism $\eta^{\prime}$. Define

$$
\widetilde{V}_{1}^{\prime}\left(\phi_{1}\left(\mathbf{s}_{1}\right) ; \phi_{1}\left(\mathbf{t}_{1}\right)\right)=\int \widetilde{V}_{1}\left(\mathbf{s}_{1} ; \mathbf{y}_{1}\right) f_{1}\left(\mathbf{y}_{1} \mid \phi_{1}\left(\mathbf{y}_{1}\right)=\phi_{1}\left(\mathbf{t}_{1}\right)\right) d \mathbf{y}_{1}
$$

and

$$
\widetilde{\xi}_{1}^{\prime}\left(\phi_{1}\left(\mathbf{t}_{1}\right)\right)=\int \widetilde{\xi}_{1}\left(\mathbf{y}_{1}\right) f_{1}\left(\mathbf{y}_{1} \mid \phi_{1}\left(\mathbf{y}_{1}\right)=\phi_{1}\left(\mathbf{t}_{1}\right)\right) d \mathbf{y}_{1}
$$

which are player 1's expected asset value and expected payment when his type is equivalent to $\mathbf{s}_{1}$ and he reports a type equivalent to $\mathbf{t}_{1}$.

The previous lemma establishes that for equivalent types $\mathbf{s}_{1}$ and $\mathbf{s}_{1}^{\prime}$ the incentive compatibility constraints yield

$$
\widetilde{V}_{1}\left(\mathbf{s}_{1} ; \mathbf{s}_{1}\right)-\widetilde{\xi}_{1}\left(\mathbf{s}_{1}\right)=\widetilde{V}_{1}\left(\mathbf{s}_{1} ; \mathbf{s}_{1}^{\prime}\right)-\widetilde{\xi}_{1}\left(\mathbf{s}_{1}^{\prime}\right)
$$


Integrating these relationships along the set of equivalent types with respect to $f_{1}\left(\mathbf{s}_{1}^{\prime} \mid \phi_{1}\left(\mathbf{s}_{1}^{\prime}\right)=\phi_{1}\left(\mathbf{s}_{1}\right)\right)$ results in

$$
\widetilde{V}_{1}\left(\mathbf{s}_{1} ; \mathbf{s}_{1}\right)-\widetilde{\xi}_{1}\left(\mathbf{s}_{1}\right)=\widetilde{V}_{1}^{\prime}\left(\phi_{1}\left(\mathbf{s}_{1}\right) ; \phi_{1}\left(\mathbf{s}_{1}\right)\right)-\widetilde{\xi}_{1}^{\prime}\left(\phi_{1}\left(\mathbf{s}_{1}\right)\right)
$$

Player 1's incentive compatibility constraint under $\eta$ for non-equivalent types $\mathbf{s}_{1}$ and $\mathbf{t}_{1}$ is

$$
\widetilde{V}_{1}\left(\mathbf{s}_{1} ; \mathbf{s}_{1}\right)-\widetilde{\xi}_{1}\left(\mathbf{s}_{1}\right) \geq \widetilde{V}_{1}\left(\mathbf{s}_{1} ; \mathbf{t}_{1}\right)-\widetilde{\xi}_{1}\left(\mathbf{t}_{1}\right) .
$$

Integrating these relationships along the set of equivalent types to $\mathbf{t}_{1}$ yields

$$
\widetilde{V}_{1}\left(\mathbf{s}_{1} ; \mathbf{s}_{1}\right)-\widetilde{\xi}_{1}\left(\mathbf{s}_{1}\right) \geq \widetilde{V}_{1}^{\prime}\left(\phi_{1}\left(\mathbf{s}_{1}\right) ; \phi_{1}\left(\mathbf{t}_{1}\right)\right)-\widetilde{\xi}_{1}^{\prime}\left(\phi_{1}\left(\mathbf{t}_{1}\right)\right) \text {. }
$$

Combining (6) and (7) yields

$$
\widetilde{V}_{1}^{\prime}\left(\phi_{1}\left(\mathbf{s}_{1}\right) ; \phi_{1}\left(\mathbf{s}_{1}\right)\right)-\widetilde{\xi}_{1}^{\prime}\left(\phi_{1}\left(\mathbf{s}_{1}\right)\right) \geq \widetilde{V}_{1}^{\prime}\left(\phi_{1}\left(\mathbf{s}_{1}\right) ; \phi_{1}\left(\mathbf{t}_{1}\right)\right)-\widetilde{\xi}_{1}^{\prime}\left(\phi_{1}\left(\mathbf{t}_{1}\right)\right) .
$$

which is the incentive compatibility constraint for player 1 under mechanism $\eta^{\prime}$.

Lemma 3. The mechanisms $\eta$ and $\eta^{\prime}$ are revenue equivalent.

Proof. The expected revenue for the seller under mechanism $\eta$ is

$$
\begin{aligned}
E R(\eta) & =\int \sum_{k} \xi_{k}(\mathbf{s}) f(\mathbf{s}) d \mathbf{s} \\
& =\sum_{k} \int \xi_{k}(\mathbf{s}) \prod f_{i}\left(\mathbf{s}_{i}\right) d \mathbf{s}_{i} \\
& =\sum_{k} \int f_{-1}\left(\mathbf{s}_{-1}\right) \int f_{\phi_{1}}(\alpha) \int \xi_{k}\left(\mathbf{s}_{1}, \mathbf{s}_{-1}\right) f_{1}\left(\mathbf{s}_{1} \mid \phi\left(\mathbf{s}_{1}\right)=\alpha\right) d \mathbf{s}_{1} d \alpha d \mathbf{s}_{-1} . \\
& =\sum_{k} \iint \xi_{k}^{\prime}\left(\alpha, \mathbf{s}_{-i}\right) f_{-1}\left(\mathbf{s}_{-1}\right) f_{\phi_{1}}(\alpha) d \alpha d \mathbf{s}_{-1} \\
& =E R\left(\eta^{\prime}\right)
\end{aligned}
$$

Proof of Theorem 1. Follows from the above three lemmas.

\section{Proof of Theorem 2}

\section{Notation}

For every $t$ in the support of $\phi\left(X_{1}, X_{2}\right)$ define:

$$
\underline{x}_{1}(t)=\inf \left\{x_{1} \mid \exists x_{2} \in\left[\underline{z}_{2}, \bar{z}_{2}\right], \phi\left(x_{1}, x_{2}\right)=t\right\}
$$

and

$$
\bar{x}_{1}(t)=\sup \left\{x_{1} \mid \exists x_{2} \in\left[\underline{z}_{2}, \bar{z}_{2}\right], \phi\left(x_{1}, x_{2}\right)=t\right\},
$$

analogously define $\underline{x}_{2}(t)$ and $\bar{x}_{2}(t)$. Note that these objects are well-defined and increasing when $\phi(\cdot, \cdot)$ is increasing.

Define implicitly for every $t, \psi_{2}(\cdot, t):\left[\underline{x}_{1}(t), \bar{x}_{1}(t)\right] \rightarrow\left[\underline{x}_{2}(t), \bar{x}_{2}(t)\right]$

$$
\phi\left(x_{1}, \psi_{2}\left(x_{1}, t\right)\right)=t
$$


and $\psi_{1}(\cdot, t):\left[\underline{x}_{2}(t), \bar{x}_{2}(t)\right] \rightarrow\left[\underline{x}_{1}(t), \bar{x}_{1}(t)\right]$

$$
\phi\left(\psi_{1}\left(x_{2}, t\right), x_{2}\right)=t
$$

Note that $\psi_{1}(\cdot, t)$ and $\psi_{2}(\cdot, t)$ are well-defined decreasing functions and $\psi_{1}^{-1}(\cdot, t)=\psi_{2}(\cdot, t)$.

$$
\frac{d}{d x_{1}} \phi\left(x_{1}, \psi_{2}\left(x_{1}, t\right)\right)=0 \Leftrightarrow \partial_{1} \phi\left(x_{1}, \psi_{2}\left(x_{1}, t\right)\right)=-\partial_{2} \phi\left(x_{1}, \psi_{2}\left(x_{1}, t\right)\right) \partial_{x_{1}} \psi_{2}\left(x_{1}, t\right)
$$

or

$$
\partial_{x_{1}} \psi_{2}\left(x_{1}, t\right)=-\frac{\partial_{1} \phi\left(x_{1}, \psi_{2}\left(x_{1}, t\right)\right)}{\partial_{2} \phi\left(x_{1}, \psi_{2}\left(x_{1}, t\right)\right)}
$$

and analogously

$$
\partial_{x_{2}} \psi_{1}\left(x_{2}, t\right)=-\frac{\partial_{2} \phi\left(\psi_{1}\left(x_{2}, t\right), x_{2}\right)}{\partial_{1} \phi\left(\psi_{1}\left(x_{2}, t\right), x_{2}\right)} .
$$

For every function $V$ which admits $\phi$ as a sufficient statistic for its first two arguments, define for all $t$ and $\mathbf{t}_{-12}$,

$$
V^{\phi}\left(t, \mathbf{t}_{-12}\right)=V\left(x_{1}, x_{2}, \mathbf{t}_{-12}\right)
$$

if there exist $x_{1}$ and $x_{2}$ such that $\phi\left(x_{1}, x_{2}\right)=t$. Note that,

$$
V^{\phi}\left(t, t_{-12}\right)=V\left(x_{1}, \psi_{2}\left(x_{1}, t\right), t_{-12}\right)
$$

and therefore

$$
\begin{aligned}
\partial_{t} V^{\phi}\left(t, t_{-12}\right) & =\partial_{2} V\left(x_{1}, \psi_{2}\left(x_{1}, t\right), t_{-12}\right) \partial_{t} \psi_{2}\left(x_{1}, t\right) \\
& =\frac{\partial_{2} V\left(x_{1}, \psi_{2}\left(x_{1}, t\right), t_{-12}\right)}{\partial_{2} \phi\left(x_{1}, \psi_{2}\left(x_{1}, t\right)\right)} .
\end{aligned}
$$

By a similar argument one can show that

$$
\partial_{t} V^{\phi}\left(t, t_{-12}\right)=\frac{\partial_{1} V\left(\psi_{1}\left(x_{2}, t\right), x_{2}, t_{-12}\right)}{\partial_{1} \phi\left(\psi_{1}\left(x_{2}, t\right), x_{2}\right)} .
$$

These relationships show that $\partial_{t} V^{\phi}$ is increasing if $V$ is increasing. Furthermore, for all $j \geq 3$

$$
\partial_{t j} V^{\phi}\left(t, t_{-1}\right)=\frac{\partial_{1 j} V\left(\psi_{1}\left(x_{2}, t\right), x_{2}, t_{-1}\right)}{\partial_{1} \phi\left(\psi_{1}\left(x_{2}, t\right), x_{2}\right)},
$$

which means that $\partial_{t j} V^{\phi}$ and $\partial_{1 j} V$ will have the same sign.

Lemma 4. The survival function and density function of $\phi\left(X_{1}, X_{2}\right)$ are given by

$$
\begin{aligned}
\bar{F}_{\phi}(t) & =\int_{-\infty}^{\infty} f_{1}(x) \bar{F}_{2}\left(\psi_{2}(x, t)\right) d x=\int_{-\infty}^{\infty} f_{2}(x) \bar{F}_{1}\left(\psi_{1}(x, t)\right) d x, \text { and } \\
f_{\phi}(t) & =\int_{-\infty}^{\infty} \frac{f_{1}(x) f_{2}\left(\psi_{2}(x, t)\right)}{\partial_{2} \phi\left(x, \psi_{2}(x, t)\right)} d x=\int_{-\infty}^{\infty} \frac{f_{2}(x) f_{1}\left(\psi_{1}(x, t)\right)}{\partial_{1} \phi\left(\psi_{1}(x, t), x\right)} d x
\end{aligned}
$$

Proof. Note that

$$
\operatorname{Pr}\left[\phi\left(X_{1}, X_{2}\right) \geq t\right]=\bar{F}_{\phi}(t)=\int \operatorname{Pr}\left[\phi\left(X_{1}, X_{2}\right) \geq t \mid X_{1}=x\right] f_{1}(x) d x .
$$


Since $X_{1}$ and $X_{2}$ are independent, we have

$$
\operatorname{Pr}\left[\phi\left(X_{1}, X_{2}\right) \geq t \mid X_{1}=x\right]=\operatorname{Pr}\left[X_{2} \geq \psi_{2}(x, t) \mid X_{1}=x\right]=\bar{F}_{2}\left(\psi_{2}(x, t)\right) .
$$

Substituting back into the integral yields the first expression. Note that

$$
\frac{d}{d t} \phi\left(x, \psi_{2}(x, t)\right)=1 \Leftrightarrow \partial_{2} \phi\left(x, \psi_{2}(x, t)\right) \partial_{t} \psi_{2}(x, t)=1
$$

or

$$
\partial_{t} \psi_{2}(x, t)=\frac{1}{\partial_{2} \phi\left(x, \psi_{2}(x, t)\right)}
$$

Then,

$$
\begin{aligned}
f_{\phi}(t) & =-\partial_{t} \bar{F}_{\phi}(t) \\
& =\int_{-\infty}^{\infty} f_{1}(x) f_{2}\left(\psi_{2}(x, t)\right) \partial_{t} \psi_{2}(x, t) d x
\end{aligned}
$$

Substituting Equation 12 yields:

$$
=\int_{-\infty}^{\infty} \frac{f_{1}(x) f_{2}\left(\psi_{2}(x, t)\right)}{\partial_{2} \phi\left(x, \psi_{2}(x, t)\right)} d x
$$

Proof of Theorem 2. For notational convenience, we consider the case where buyer 1 and 2's information is centralized under the control of a new buyer $c$. Let

$$
A=\left\{A_{1}, A_{2}, . ., A_{n}\right\}
$$

and

$$
A^{\prime}=\left\{A_{c}, A_{3}^{\prime}, . ., A_{n}^{\prime}\right\}
$$

where $A_{c}=A_{1} \cup A_{2}$ and $A_{i}=A_{i}^{\prime}$ for all $i \geq 3$. Under our assumptions every buyer's information can be summarized by $T_{i}=\phi_{i}^{A}\left(\mathbf{S}_{i}\right)$ and in the optimal scalar mechanism $\mu^{A}$ for information profile $A$ only reports $t_{i}$ are required from buyers. In the concentrated environment $A^{\prime}$ a scalar mechanism will require reports $t_{i}=T_{i}$ from buyers 3 through $n$, while it will ask buyer $c$ to submit a report $t_{c}=\phi\left(T_{1}, T_{2}\right)$. Denote by $f_{i}\left(t_{i}\right), F_{i}\left(t_{i}\right)$ and $\left[\underline{a}_{i}, \bar{a}_{i}\right]$ the density, distribution function and respectively support of the random variables $T_{i}$. Further, denote by $f_{\phi}(t)$ and $F_{\phi}(t)$ the density and distribution function of the random variable $\phi\left(T_{1}, T_{2}\right)$. Denote by $t_{i}$ and $t_{-i j}$ the vector of reports excluding buyer $i$ or buyers $i$ and $j$.

Define for all $t_{c}$,

$$
V^{A^{\prime}}\left(t_{c}, t_{-12}\right)=V^{A}\left(t_{1}, t_{2}, t_{-12}\right)
$$

if there exist $t_{1}$ and $t_{2}$ such that $\phi\left(t_{1}, t_{2}\right)=t$. From the definition of $\psi_{2}(\cdot)$, we also have

$$
V^{A^{\prime}}\left(t_{c}, t_{-12}\right)=V^{A}\left(t_{1}, \psi_{2}\left(t_{1}, t_{c}\right), t_{-12}\right)
$$

and therefore

$$
\begin{aligned}
\partial_{t_{c}} V^{A^{\prime}}\left(t_{c}, t_{-12}\right) & =\partial_{2} V^{A}\left(t_{1}, \psi_{2}\left(t_{1}, t_{c}\right), t_{-12}\right) \partial_{t_{c}} \psi_{2}\left(t_{1}, t_{c}\right) \\
& =\frac{\partial_{2} V^{A}\left(t_{1}, \psi_{2}\left(t_{1}, t_{c}\right), t_{-12}\right)}{\partial_{2} \phi\left(t_{1}, \psi_{2}\left(t_{1}, t_{c}\right)\right)}
\end{aligned}
$$


Where the last equality follows from Equation 12 . By a similar argument, one can show that

$$
\partial_{t_{c}} V^{A^{\prime}}\left(t_{c}, t_{-12}\right)=\frac{\partial_{1} V^{A}\left(\psi_{1}\left(t_{2}, t_{c}\right), t_{2}, t_{-12}\right)}{\partial_{1} \phi\left(\psi_{1}\left(t_{2}, t_{c}\right), t_{2}\right)}
$$

Consider the virtual valuation of player $c$,

$$
H_{c}^{A^{\prime}}\left(t_{c}, t_{-12}\right)=V^{A^{\prime}}\left(t_{c}, t_{-12}\right)-\frac{\bar{F}_{\phi}\left(t_{c}\right)}{f_{\phi}(t)} \partial_{t_{c}} V^{A^{\prime}}\left(t_{c}, t_{-12}\right) .
$$

Under the regularity assumption $H_{c}^{A^{\prime}}\left(t, t_{-12}\right)$ is increasing in $t$.

For any mechanism $\eta=\left(p_{i}^{\prime}, \xi_{i}^{\prime}\right)_{i \in\{c, 3, . ., n\}}$ in environment $A^{\prime}$, the seller's revenue is

$$
R^{A^{\prime}}(\eta)=\int\left(\sum_{i \geq 3} p_{i}^{\prime}\left(t_{i}, t_{-i}\right) H_{i}^{A^{\prime}}\left(t_{i}, t_{-i}\right)+p_{c}^{\prime}\left(t_{c}, t_{-12}\right) H_{c}^{A^{\prime}}\left(t_{c}, t_{-12}\right)+p_{0}^{\prime} v_{0}\right) f_{\phi}\left(t_{c}\right) f\left(t_{-12}\right) d t_{c} d t_{-12} .
$$

Point-by-point maximization of the integrand yields the optimal solution $\mu^{A^{\prime}}$ where

$$
p_{i}^{\prime}\left(t_{i}, t_{-i}\right)=1 \Leftrightarrow H_{i}^{A^{\prime}}\left(t_{i}, t_{-i}\right) \geq \max _{j \neq i}\left(v_{0}, H_{c}^{A^{\prime}}\left(t_{c}, t_{-12}\right), H_{j}^{A^{\prime}}\left(t_{j}, t_{-j}\right)\right)
$$

and zero otherwise. Since the functions $H_{i}^{A^{\prime}}$ are monotonic, $H_{i}^{A^{\prime}}\left(t_{i}, t_{-i}\right) \geq v_{0}$ implies $H_{i}^{A^{\prime}}\left(t_{i}^{\prime}, t_{-i}\right) \geq v_{0}$ for all $t_{i}^{\prime} \geq t_{i}$. Furthermore, for all $j$, we have

$$
H_{i}^{A^{\prime}}\left(t_{i}, t_{-i}\right) \geq H_{j}^{A^{\prime}}\left(t_{i}, t_{-i}\right) \Leftrightarrow g_{i}^{A^{\prime}}\left(t_{i}, t_{-i}\right) \leq g_{j}^{A^{\prime}}\left(t_{i}, t_{-i}\right)
$$

and for all $t_{i}^{\prime} \geq t_{i}$

$$
g_{i}^{A^{\prime}}\left(t_{i}^{\prime}, t_{-i}\right) \leq g_{i}^{A^{\prime}}\left(t_{i}, t_{-i}\right) \leq g_{j}^{A^{\prime}}\left(t_{i}, t_{-i}\right) \leq g_{j}^{A^{\prime}}\left(t_{i}^{\prime}, t_{-i}\right)
$$

which means

$$
H_{i}^{A^{\prime}}\left(t_{i}^{\prime}, t_{-i}\right) \geq H_{j}^{A^{\prime}}\left(t_{i}^{\prime}, t_{-i}\right)
$$

In particular, the allocation probabilities $p_{i}^{\prime}\left(t_{i}, t_{-i}\right)$ are monotone in $t_{i}$, which, in turn, is a sufficient condition for implementation (Myerson 1981).

For bidder $c$ we have

$$
p_{c}^{\prime}\left(t_{c}, t_{-12}\right)=1 \Leftrightarrow H_{c}^{A^{\prime}}\left(t_{c}, t_{-12}\right) \geq \max _{i}\left(v_{0}, H_{i}^{A^{\prime}}\left(t_{i}, t_{-i}\right)\right)
$$

and zero otherwise. An argument similar to the above suggests that for any $t_{-12}$ and $v_{0}$, the set of types for which bidder $c$ gets the object is given by

$$
M_{c}=\left\{\left(t_{1}, t_{2}\right) \mid \phi\left(t_{1}, t_{2}\right)=t_{c} \geq \tau\left(t_{-12}, v_{0}\right)\right\} .
$$

for some function, $\tau$. The expected payment received by the auctioneer from bidder $c$ is therefore,

$$
\xi_{c}^{A^{\prime}}=\int\left(\int_{t_{c}>\tau\left(t_{-12}, v_{0}\right)} H_{c}^{A^{\prime}}\left(t_{c}, t_{-12}\right) f_{\phi}\left(t_{c}\right) d t_{c}\right) f\left(t_{-12}\right) d t_{-12}
$$

Define

$$
Q^{\prime}(t)=\int_{t_{c}>t} H_{c}^{A^{\prime}}\left(t_{c}, t_{-12}\right) f_{\phi}\left(t_{c}\right) d t_{c}
$$


then

$$
\xi_{c}^{A^{\prime}}=\int Q^{\prime}\left(\tau\left(t_{-12}, v_{0}\right)\right) f\left(t_{-12}\right) d t_{-12} .
$$

Consider a mechanism $\omega=\left(p_{i}, \xi_{i}\right)_{i \in\{1, . ., n\}}$ for the environment $A$, with the following properties $p_{1}\left(t_{1}, t_{-1}\right) \equiv$ $0, p_{2}\left(t_{1}, t_{2}, t_{-12}\right) \equiv p_{c}^{\prime}\left(\phi\left(t_{1}, t_{2}\right), t_{-12}\right)$ and $p_{i}\left(t_{i}, t_{-i}\right) \equiv p_{i}^{\prime}\left(t_{i}, t_{-i}\right)$ for all $i \geq 3$. The mechanism $\omega$ is incentive compatible since all the functions are monotone in own type 7 Furthermore, the expected payment received from bidders 3 through $n$ is the same under $\omega$ and $\mu^{A^{\prime}}$.

The expected payment from bidder 2 in this case is therefore

$$
\xi_{2}^{A}=\int\left(\iint_{M_{c}} H_{2}^{A}\left(t_{1}, t_{2}, t_{-12}\right) f_{1}\left(t_{1}\right) f_{2}\left(t_{2}\right) d t_{1} d t_{2}\right) f\left(t_{-12}\right) d t_{-12} .
$$

Define

$$
Q(t)=\iint_{\phi\left(t_{1}, t_{2}\right) \geq t} H_{2}^{A}\left(t_{1}, t_{2}, t_{-12}\right) f_{1}\left(t_{1}\right) f_{2}\left(t_{2}\right) d t_{1} d t_{2}
$$

then

$$
\xi_{2}^{A}=\int\left(Q\left(\tau\left(t_{-12}, v_{0}\right)\right)\right) f\left(t_{-12}\right) d t_{-12}
$$

In particular, if for all $t$

$$
Q^{\prime}(t)=Q(t)
$$

the expected payments of bidder $c$ under $\mu^{A^{\prime}}$ and those of bidder 2 under $\omega$ coincide, which makes the two mechanisms revenue equivalent. In equations $(8)$ and (9), we defined $\underline{x}_{1}(t)$ and $\bar{x}_{1}(t)$ for the elementary random variables, $X_{i}$. We define an identical notion for the random variables of the sufficient statistics, $T_{i}$ :

$$
\underline{t}_{1}(t)=\inf \left\{t_{1} \mid \exists t_{2}, \phi\left(t_{1}, t_{2}\right)=t\right\}
$$

and

$$
\bar{t}_{1}(t)=\sup \left\{t_{1} \mid \exists t_{2}, \phi\left(t_{1}, t_{2}\right)=t\right\},
$$

Note that $Q(t)$ may be expressed as

$$
Q(t)=\iint_{\phi\left(t_{1}, t_{2}\right) \geq t}\left(V^{A}\left(t_{1}, t_{2}, t_{-12}\right)-\frac{\bar{F}_{2}\left(t_{2}\right)}{f_{2}\left(t_{2}\right)} \partial_{2} V^{A}\left(t_{1}, t_{2}, t_{-12}\right)\right) f_{1}\left(t_{1}\right) f_{2}\left(t_{2}\right) d t_{1} d t_{2}
$$

We consider each component separately. First,

$$
\iint_{\phi\left(t_{1}, t_{2}\right) \geq t} V^{A}\left(t_{1}, t_{2}, t_{-12}\right) f_{1}\left(t_{1}\right) f_{2}\left(t_{2}\right) d t_{1} d t_{2}=\int_{\underline{t}_{1}(t)}^{\bar{t}_{1}(t)} \int_{t_{2} \geq \psi_{2}\left(t_{1}, t\right)} V^{A}\left(t_{1}, t_{2}, t_{-12}\right) f_{1}\left(t_{1}\right) f_{2}\left(t_{2}\right) d t_{2} d t_{1}
$$

Fix $t_{1}$ and introduce the change in variable

$$
t_{2}=\psi_{2}\left(t_{1}, t_{c}\right)
$$

Note that

$$
d t_{2}=\partial_{t_{c}} \psi_{2}\left(t_{1}, t_{c}\right) d t_{c}
$$

\footnotetext{
${ }^{7}$ Bidder 1's payment will be zero.
} 
The integral becomes

$$
\begin{aligned}
& \int_{\underline{t}_{1}(t)}^{\bar{t}_{1}(t)} \int_{t_{c} \geq t} V^{A}\left(t_{1}, \psi_{2}\left(t_{1}, t_{c}\right), t_{-12}\right) f_{1}\left(t_{1}\right) f_{2}\left(\psi_{2}\left(t_{1}, t_{c}\right)\right) \partial_{t_{c}} \psi_{2}\left(t_{1}, t_{c}\right) d t_{c} d t_{1} \\
= & \int_{\underline{t}_{1}(t)}^{\bar{t}_{1}(t)} \int_{t_{c} \geq t} \frac{V^{A}\left(t_{1}, \psi_{2}\left(t_{1}, t_{c}\right), t_{-12}\right) f_{1}\left(t_{1}\right) f_{2}\left(\psi_{2}\left(t_{1}, t_{c}\right)\right)}{\partial_{2} \phi\left(t_{1}, \psi_{2}\left(t_{1}, t_{c}\right)\right)} d t_{c} d t_{1} \\
= & \int_{t_{c} \geq t} \int_{\underline{t}_{1}(t)}^{\bar{t}_{1}(t)} \frac{V^{A^{\prime}}\left(t_{c}, t_{-12}\right) f_{1}\left(t_{1}\right) f_{2}\left(\psi_{2}\left(t_{1}, t_{c}\right)\right)}{\partial_{2} \phi\left(t_{1}, \psi_{2}\left(t_{1}, t_{c}\right)\right)} d t_{1} d t_{c} \\
= & \int_{t_{c} \geq t} V^{A^{\prime}}\left(t_{c}, t_{-12}\right) \int_{\underline{t}_{1}(t)}^{\bar{t}_{1}(t)} \frac{f_{1}\left(t_{1}\right) f_{2}\left(\psi_{2}\left(t_{1}, t_{c}\right)\right)}{\partial_{2} \phi\left(t_{1}, \psi_{2}\left(t_{1}, t_{c}\right)\right)} d t_{1} d t_{c} \\
= & \int_{t_{c} \geq t} V^{A^{\prime}\left(t_{c}, t_{-12}\right) f_{\phi}(t) d t_{c} .}
\end{aligned}
$$

Where the first equality follows from Equation (12), the second from (13) and Fubini's theorem, and the last by Lemma 4 .

The second part of the integral in 17 is

$$
\begin{aligned}
& -\int_{\phi\left(t_{1}, t_{2}\right) \geq t}\left(\frac{\bar{F}_{2}\left(t_{2}\right)}{f_{2}\left(t_{2}\right)} \partial_{2} V^{A}\left(t_{1}, t_{2}, t_{-12}\right)\right) f_{1}\left(t_{1}\right) f_{2}\left(t_{2}\right) d t_{1} d t_{2} \\
= & -\iint_{\phi\left(t_{1}, t_{2}\right) \geq t} f_{1}\left(t_{1}\right) \partial_{2} V^{A}\left(t_{1}, t_{2}, t_{-12}\right) \bar{F}_{2}\left(t_{2}\right) d t_{1} d t_{2} \\
= & -\int_{\underline{t}_{1}(t)}^{\bar{t}_{1}(t)} \int_{t_{2} \geq \psi_{2}\left(t_{1}, t\right)} f_{1}\left(t_{1}\right) \partial_{2} V^{A}\left(t_{1}, t_{2}, t_{-12}\right) \bar{F}_{2}\left(t_{2}\right) d t_{1} d t_{2}
\end{aligned}
$$

By a change of variables according to $t_{2}=\psi_{2}\left(t_{1}, t_{c}\right)$, the integral becomes

$$
\begin{aligned}
& =-\int_{\underline{t}_{1}(t)}^{\bar{t}_{1}(t)} \int_{t_{c} \geq t} f_{1}\left(t_{1}\right) \partial_{2} V^{A}\left(t_{1}, \psi_{2}\left(t_{1}, t_{c}\right), t_{-12}\right) \bar{F}_{2}\left(\psi_{2}\left(t_{1}, t_{c}\right)\right) \partial_{t_{c}} \psi_{2}\left(t_{1}, t_{c}\right) d t_{c} d t_{1} \\
& =-\int_{\underline{t}_{1}(t)}^{\bar{t}_{1}(t)} \int_{t_{c} \geq t} \frac{\partial_{2} V^{A}\left(t_{1}, \psi_{2}\left(t_{1}, t_{c}\right), t_{-12}\right)}{\partial_{2} \phi\left(t_{1}, \psi_{2}\left(t_{1}, t_{c}\right)\right)} f_{1}\left(t_{1}\right) \bar{F}_{2}\left(\psi_{2}\left(t_{1}, t_{c}\right)\right) d t_{c} d t_{1}
\end{aligned}
$$

Reversing the order of integration and substituting Equation (14) yields

$$
\begin{aligned}
& =-\int_{t_{c} \geq t} \int_{\underline{t}_{1}(t)}^{\bar{t}_{1}(t)} \partial_{t_{c}} V^{A^{\prime}}\left(t_{c}, t_{-12}\right) f_{1}\left(t_{1}\right) \bar{F}_{2}\left(\psi_{2}\left(t_{1}, t_{c}\right)\right) d t_{1} d t_{c} \\
& =-\int_{t_{c} \geq t} \partial_{t_{c}} V^{A^{\prime}}\left(t_{c}, t_{-12}\right) \int_{\underline{t}_{1}(t)}^{\bar{t}_{1}(t)} f_{1}\left(t_{1}\right) \bar{F}_{2}\left(\psi_{2}\left(t_{1}, t_{c}\right)\right) d t_{1} d t_{c} \\
& =-\int_{t_{c} \geq t} \partial_{t_{c}} V^{A^{\prime}}\left(t_{c}, t_{-12}\right) \bar{F}_{\phi}\left(t_{c}\right) d t_{c}
\end{aligned}
$$


Combining the two results we have

$$
\begin{aligned}
Q(t) & =\int_{t_{c} \geq t}\left(V^{A^{\prime}}\left(t_{c}, t_{-12}\right) f_{\phi}(t)-\partial_{t_{c}} V^{A^{\prime}}\left(t_{c}, t_{-12}\right) \bar{F}_{\phi}\left(t_{c}\right)\right) d t_{c} \\
& =\int_{t_{c} \geq t}\left(V^{A^{\prime}}\left(t_{c}, t_{-12}\right)-\frac{\bar{F}_{\phi}\left(t_{c}\right)}{f_{\phi}(t)} \partial_{t_{c}} V^{A^{\prime}}\left(t_{c}, t_{-12}\right)\right) f_{\phi}\left(t_{c}\right) d t_{c} \\
& =\int_{t_{c}>t} H_{c}^{A^{\prime}}\left(t_{c}, t_{-12}\right) f_{\phi}\left(t_{c}\right) d t_{c} \\
& =Q^{\prime}(t)
\end{aligned}
$$

which means that $\omega$ and $\mu^{A^{\prime}}$ generate the same revenue. However, under $\omega$, buyer 1 receives the good with probability zero. If the optimal mechanism in environment $A$ allocates to buyer 1 with positive probability, then it is, by definition, revenue superior to $\omega$ and hence to $\mu^{A^{\prime}}$. 